\title{
Chromatographic Length Separation and Photoluminescence Study on DNA-Wrapped Single-Wall and Double-Wall Carbon Nanotubes
}

\author{
Yuki Asada, Toshiki Sugai, Ryo Kitaura, and Hisanori Shinohara \\ Department of Chemistry and Institute for Advanced Research, Nagoya University, Nagoya 464-8602, Japan \\ Correspondence should be addressed to Hisanori Shinohara, noris@nagoya-u.jp
}

Received 11 May 2009; Accepted 29 July 2009

Recommended by Donglu Shi

Water-soluble DNA-wrapped single-wall and double-wall carbon nanotubes (DNA-SWNTs, DNA-DWNTs) have been well separated by length incorporating size-exclusion high-performance liquid chromatography (HPLC). The morphology and electronic properties of the size- (length-) separated DNA-SWNTs and -DWNTs are investigated by atomic force microscopy (AFM), photoluminescence (PL), and Raman spectroscopy. By using length-separated DNA-SWNTs and -DWNTs, we have found that PL intensity of the DNA-SWNTs varies sensitively depending not only on the chirality (or diameter) but more importantly on the length of the hybrids.

Copyright (C) 2009 Yuki Asada et al. This is an open access article distributed under the Creative Commons Attribution License, which permits unrestricted use, distribution, and reproduction in any medium, provided the original work is properly cited.

\section{Introduction}

Carbon nanotubes (CNTs) have attracted much attention for their unique mechanical and electronic properties, which have led to some novel applications [1-3]. Above all, double-wall carbon nanotubes (DWNTs) $[4,5]$ have attracted much attention because of their unique physical and chemical properties compared to those of singlewall carbon nanotubes (SWNTs) and multiwall carbon nanotubes (MWNTs). Since DWNTs have been applied as channels of nanodevices [6], separation and purification of DWNTs are required for obtaining the DWNTs of much better quality. CNTs are, however, generally obtained as a mixture of various structures of CNTs. Furthermore, CNTs generally form bundles as mixtures, so that it has been difficult to obtain individual CNTs. Therefore, complete dispersion [7-9] and separation of individual CNTs are of considerable importance to investigate the inherent structural and electronic properties of CNTs.

During the past several years, surfactants and polymers have been used to prepare water-soluble and individually dispersed SWNTs. The findings by O'Connell et al., who have observed structured near-infrared photoluminescence (PL) from suitably isolated semiconducting SWNTs [7], allow us to apply SWNTs to wide range of optical characterization [10] as well as chemical and biological applications [11]. Moreover, progress has been made in the separation of SWNTs by band gap [11-14], diameter [15, 16], and length [17-19].

Zheng and coworkers are the first to demonstrate that SWNTs can well be separated and become water-soluble SWNTs by using DNA. DNA-wrapped single-wall carbon nanotubes (DNA-SWNTs) are known to have a high solubility and stability in water due to the $\pi$ - $\pi$ interaction between aromatic bases of DNA and SWNTs $[9,14,20]$. This $\pi-\pi$ interaction has been found to be crucial for individually dispersing and separating SWNTs by using highperformance liquid chromatography (HPLC) [14, 18]. Based on this progress, various fundamental and applied studies have been carried out on DNA-SWNT hybrid materials.

Recently, we have reported the synthesis and spectroscopic characterization of isolated DNA-SWNTs by using natural DNA from salmon sperm in aqueous solution [9]. Here, we report the synthesis and separation of DNASWNTs and DNA-DWNTs by length incorporating size exclusion chromatography (SEC) with newly developed special columns. The CNTs separated are characterized and investigated by atomic force microscopy (AFM), PL, and 


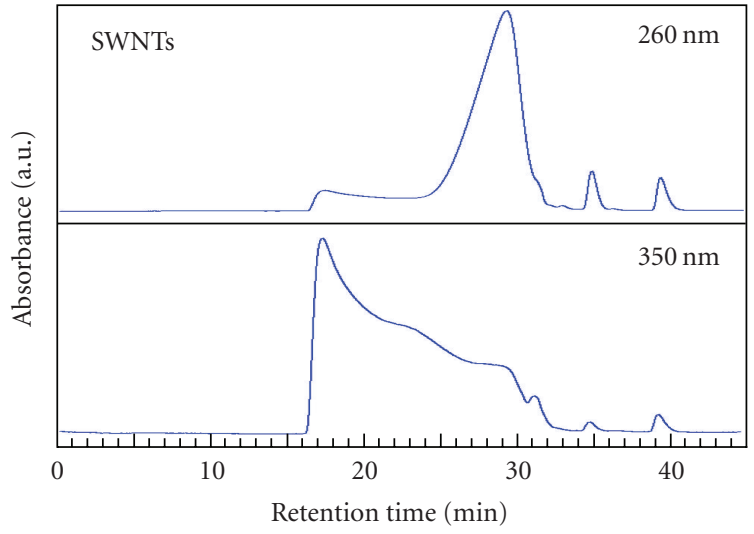

(a)

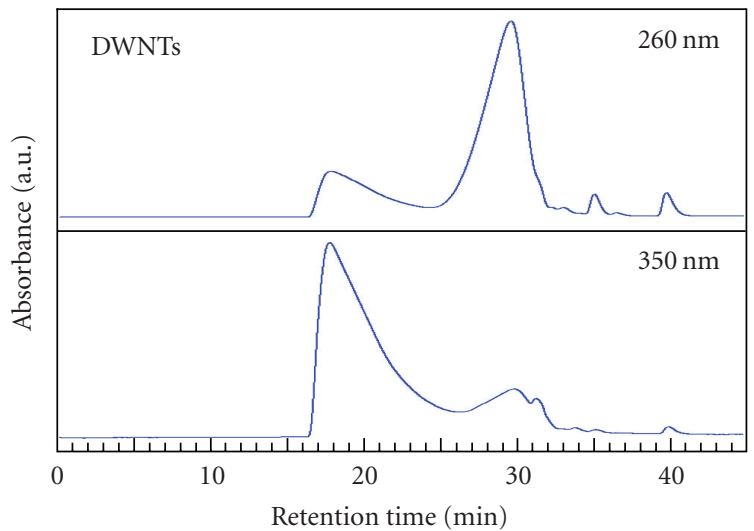

(b)

FIGURE 1: A typical chromatogram of size exclusion column separation of (a) DNA-SWNTs and (b) DNA-DWNTs. The detection UV wavelengths used in the column separation were 260 and $350 \mathrm{~nm}$. The flow rate and injection volume were $1.0 \mathrm{~mL} / \mathrm{min}$ and $1 \mathrm{~mL}$, respectively.

Raman spectroscopy to obtain length-dependent optical properties of DNA-CNTs.

\section{Experimental}

In our previous study, we reported the synthesis of watersoluble DNA-SWNTs by using DNA from salmon. In the present study, we used three different kinds of CNTs which are purified HiPco SWNTs [21], CoMoCAT SWNTs (CoMo catalyst) [22], and DWNTs (TORAY Inc.). The purity of DWNTs (against residual SWNTs and MWNTs) is more than ca. $90 \%$, and the average diameters of the outer and inner tubes of the DWNTs are 1.4 and $0.7 \mathrm{~nm}$, respectively, based on high-resolution TEM (HRTEM) observations.

Length separation by HPLC was carried out by using sizeexclusion columns: COSMOSIL CNT $(7.5 \mathrm{~mm} \times 300 \mathrm{~mm}$, nacalai tesque Inc., a newly developed column through the Nagoya University group-nacalai collaboration) and Sepax CNT $(7.5 \mathrm{~mm} \times 250 \mathrm{~mm}$, Sepax Technologies, Inc.). Similar to our previous study $[19,23]$, we used three types of columns (pore size: 300,1000 , and $2000 \AA$ ) to separate both shorter and longer tubes at one time. Samples were filtrated through a $0.25 \mu \mathrm{m}$ filter and injected in $1.0 \mathrm{~mL}$ at $\mathrm{pH} 7 \mathrm{buffer}$ containing $40 \mathrm{mM}$ Tris, $0.5 \mathrm{mM}$ EDTA, and $0.2 \mathrm{M} \mathrm{NaCl}$. Each fraction was collected at every 1 minute intervals and characterized by AFM to obtain the length distribution of CNTs separated.

Atomic force microscopy (AFM) was used for observing structures of the DNA-CNTs and for determining the lengths and morphology of the SWNTs and DWNTs. The samples were deposited onto a silanized mica (AP-mica), rinsed with MilliQ water, and dried with nitrogen gas. AP-mica was prepared as follows: freshly cleaved mica, $10 \mu \mathrm{L}$ of 3aminopropyltriethoxy silane (APTES), and $5 \mu \mathrm{L}$ of N, Ndiisopropylethylamine were kept in a desiccator filled with Ar gas. APTES was removed from the desiccator after 1 hour reaction, whereas the mica was maintained in the desiccator for 24 hours. After rinsing the surface of the so-prepared APmica by MilliQ water, it was dried with $\mathrm{N}_{2}$ gas. Topographic imaging of DNA-CNTs was obtained by operating the AFM in a tapping mode with a Dimension 3100, Nanoscope IV (Veeco, Digital Instruments).

The PL measurements were performed on a Shimadzu NIR-PL system (Shimadzu CNT-RF), and UV-vis-NIR absorption spectra were measured on a JASCO V-570 spectrophotometer.

The dried samples for Raman measurements were prepared by dropping the DNA-SWNTs solution onto a $\mathrm{SiO}_{2}$ substrate and then dried using an electric heater. Raman spectra were measured by using a Horiba Jobin Yvon HR-800 spectrometer.

For optical measurements, the samples were freeze dried to replace water with $\mathrm{D}_{2} \mathrm{O}$. As a comparison, SWNTs were also dispersed with $1 \%$ sodium dodecyl sulphate (SDS) in $\mathrm{D}_{2} \mathrm{O}$ and sonicated for 30,60 , and 120 minutes. The suspensions were centrifuged to obtain isolated SDS-dispersedSWNTs (SDS-SWNTs). The $\mathrm{pH}$ of the resultant supernatants was kept at $\mathrm{pH} 8.0$ by adding a suitable amount of $\mathrm{NaOH}$ aq (in $\mathrm{D}_{2} \mathrm{O}$ ).

\section{Results and Discussion}

\subsection{Comparison of DWNTs with SWNTs}

3.1.1. Length Separation of DNA-DWNTs by High-Performance Liquid Chromatography. The HPLC separation of the samples (CoMoCAT-SWNTs and -DWNTs) was performed by COSMOSIL CNT columns. Figure 1 is typical HPLC chromatograms of DNA-SWNTs (CoMoCAT) and DNADWNTs, where the detecting UV wavelengths at 260 and $350 \mathrm{~nm}$ are used. The chromatogram shows two distinct fractions corresponding to free (intact) DNA and the DNACNTs. The elution of free DNA observed at $260 \mathrm{~nm}$ has a maximum at 30 minutes and that of DNA-SWNTs observed at $350 \mathrm{~nm}$ continues from 17 to 30 minutes. The overall HPLC profile changes by sonication time employed, and the intensity of the latter fraction increases as the sonication time increases. 


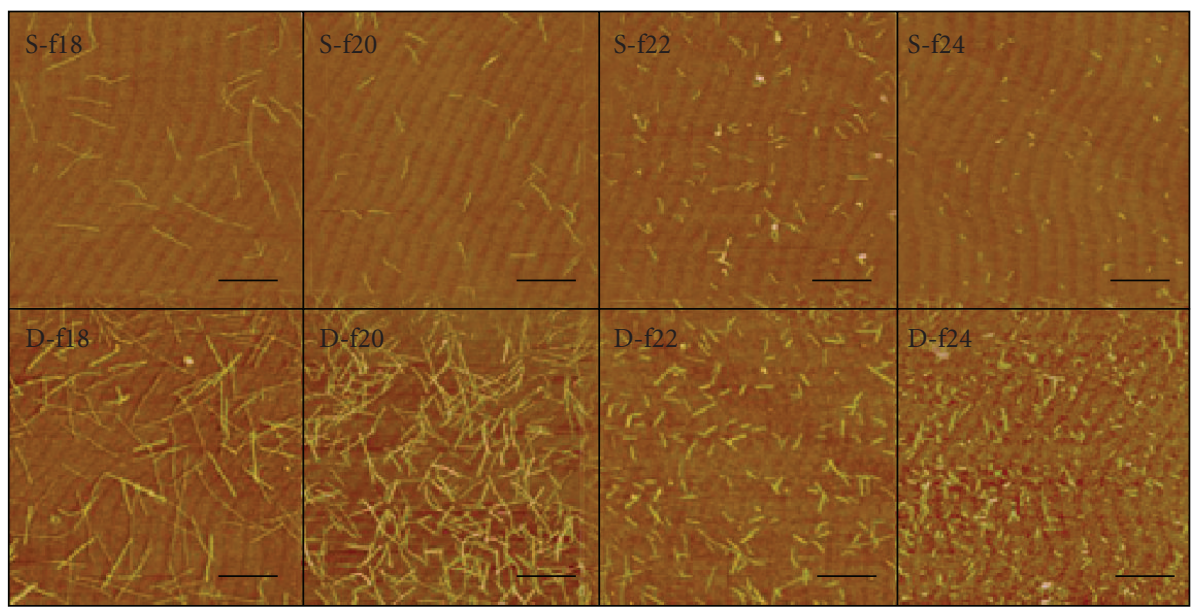

FIGURE 2: AFM images taken with $\mathrm{f} 18, \mathrm{f} 20, \mathrm{f} 22$, and $\mathrm{f} 24$ fractions of DNA-SWNTs (S) and DNA-DWNTs (D). The scale bars shown in the images are $500 \mathrm{~nm}$.

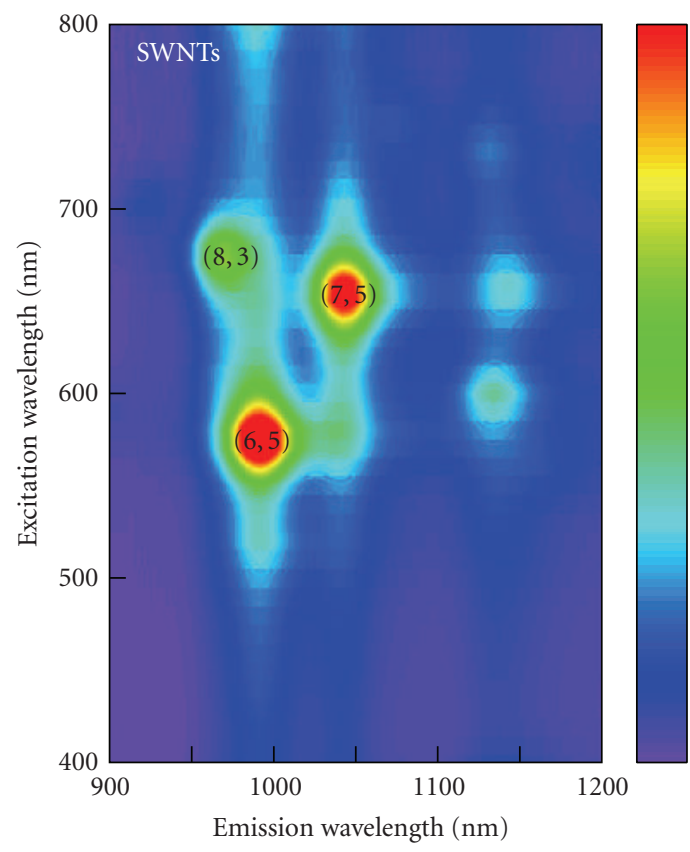

(a)

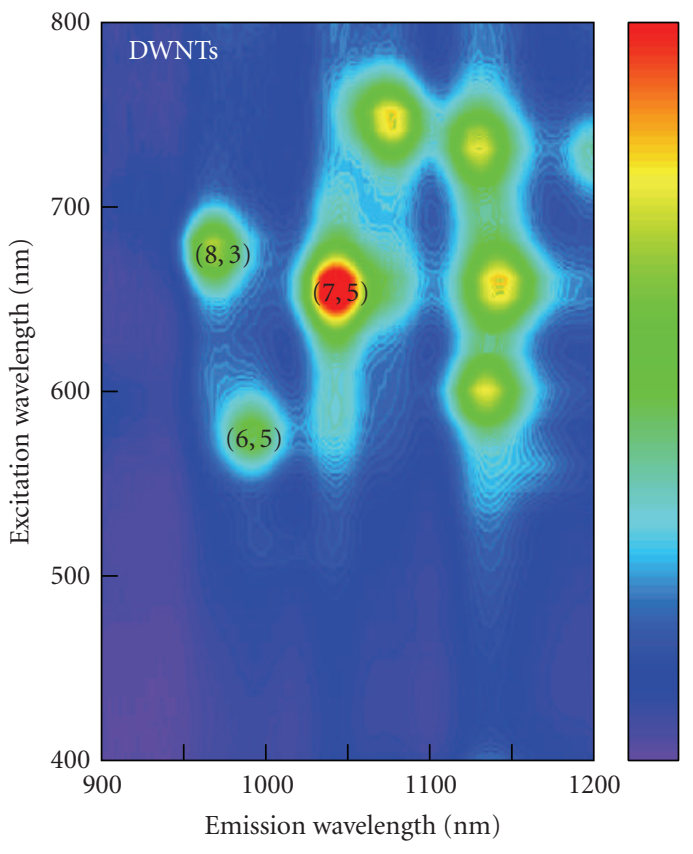

(b)

Figure 3: PL maps of DNA-SWNTs and DNA-DWNTs.

The separation by length was confirmed by AFM observations on the separated fractions as shown in Figure 2. It is known that AP-mica surfaces have relatively strong interactions with DNA and that the DNA-CNTs are easily observable on the surface by AFM. With the present HPLC column, the length of both DNA-SWNTs and DNA-DWNTs decreases as the elution (retention) time increases. Although images of only four HPLC fractions from f18 to $\mathrm{f} 24$ are shown here, we have also obtained much shorter SWNTs/DWNTs by the present separation. The results of the HPLC separation by length as revealed by the AFM measurements are summarized in Table 1.
TABLE 1: Average length of DNA-SWNTs and DNA-DWNTs after size exclusion chromatography separation.

\begin{tabular}{lcccc}
\hline \multicolumn{5}{c}{ Average length/nm } \\
\hline & $\mathrm{f} 18$ & $\mathrm{f} 20$ & $\mathrm{f} 22$ & $\mathrm{f} 24$ \\
\hline SWNTs & $393 \pm 87$ & $199 \pm 49$ & $109 \pm 25$ & $52 \pm 21$ \\
DWNTs & $378 \pm 54$ & $202 \pm 34$ & $100 \pm 20$ & $51 \pm 15$ \\
\hline
\end{tabular}

CoMoCAT-SWNTs have smaller diameter distribution than that of the DWNTs used in the present study. However, no significant difference in retention times between SWNTs and DWNTs can be observed at each fraction, indicating 


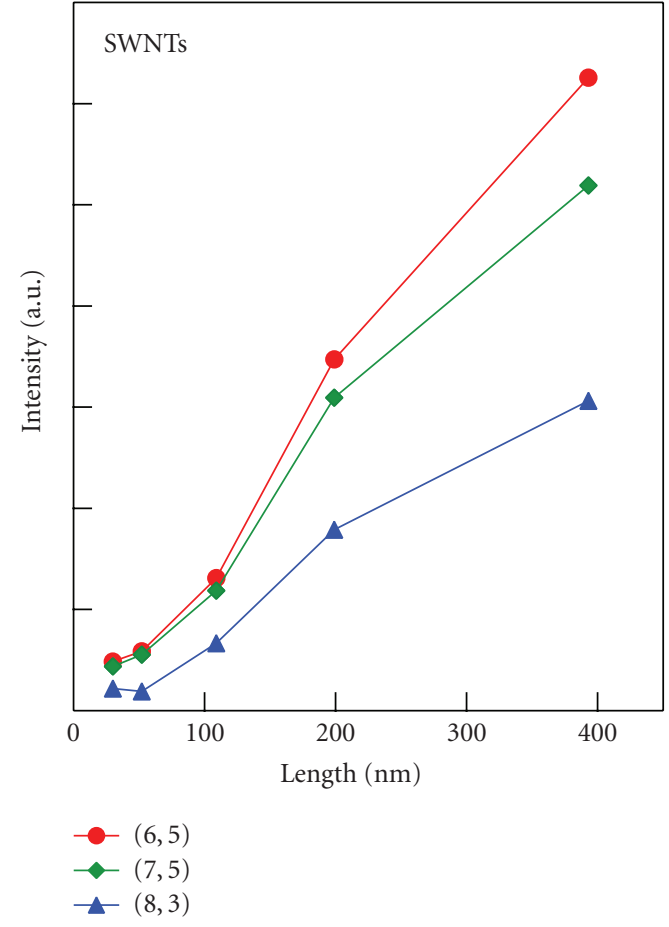

(a)

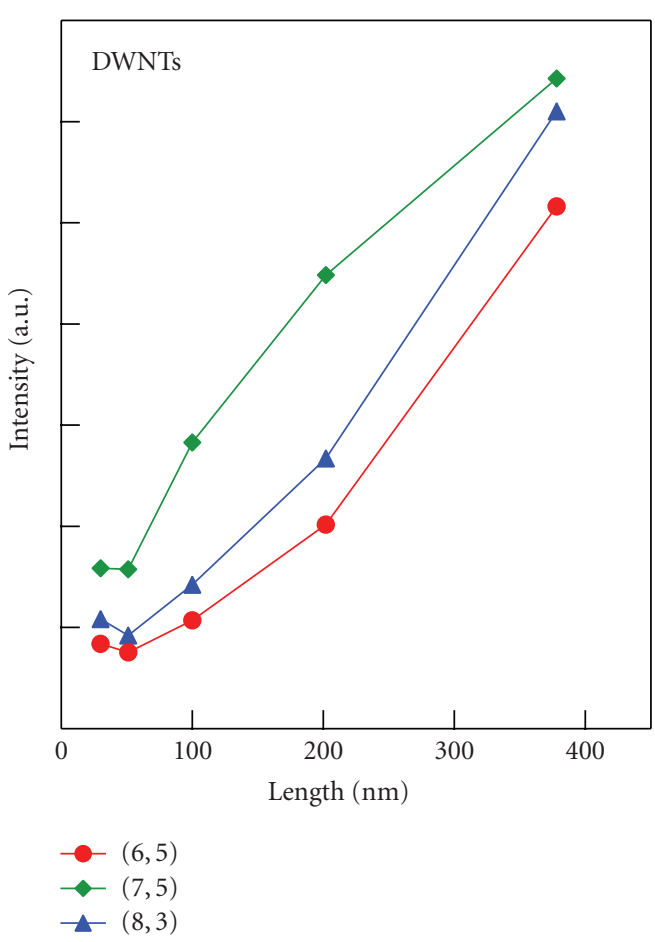

(b)

Figure 4: Length dependence of the PL intensities of DNA-SWNTs and DNA-DWNTs.

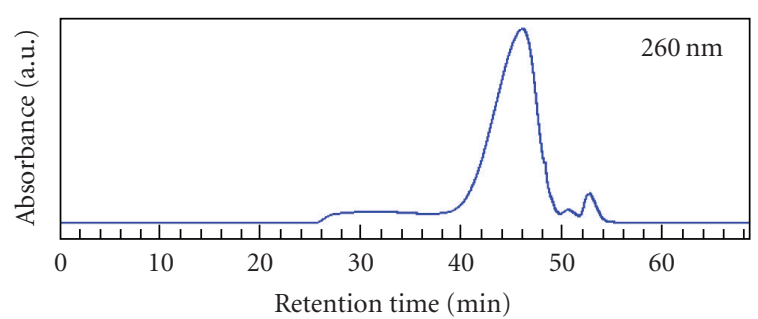

(a)

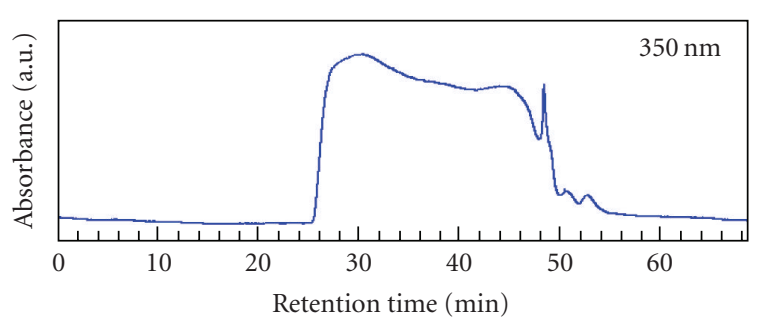

(b)

FIGURE 5: A typical chromatogram of SEC separation of DNASWNTs (HiPco). The detection UV wavelengths used in the column separation were 260 and $350 \mathrm{~nm}$. The flow rate and injection volume were $1.0 \mathrm{~mL} / \mathrm{min}$ and $1 \mathrm{~mL}$, respectively.

that the SEC columns employed here can separate and recognize SWNTs/DWNTs by length, but not by diameter or chirality.

Based on a statistical analysis of the AFM results on SWNTs/DWNTs, we have obtained the following empirical

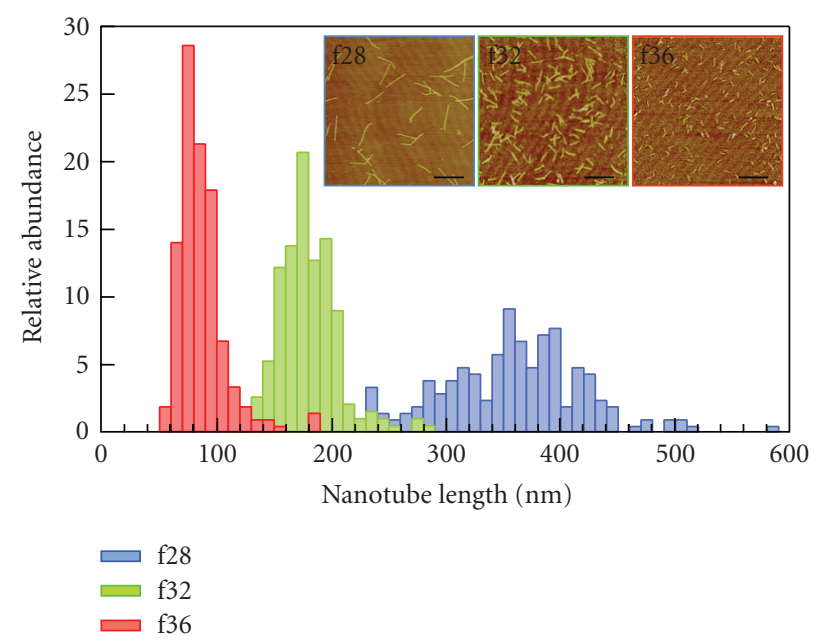

Figure 6: Histogram of length distribution of f28 (blue), f32 (green), and f36 (red) fractions. The insert shows the AFM images of each fraction. The scale bars are $500 \mathrm{~nm}$.

equation:

$$
\log (L)=-0.14 t+5.11,
$$

where $L$ is the length (nm) of SWNTs/DWNTs and $t$ is retention time (minute). We have found that this equation can be applied to both SWNTs and DWNTs having wide length distributions.

3.1.2. Photoluminescence Measurements on DNA-SWNTs and DNA-DWNTs. Figure 3 is typical PL contour plots of (a) 


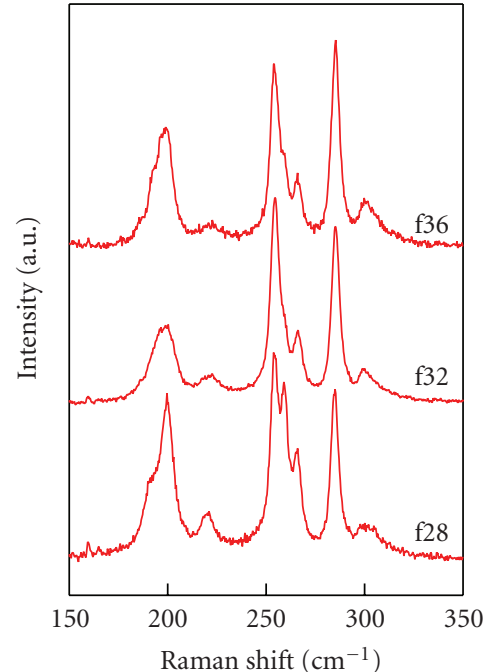

(a)

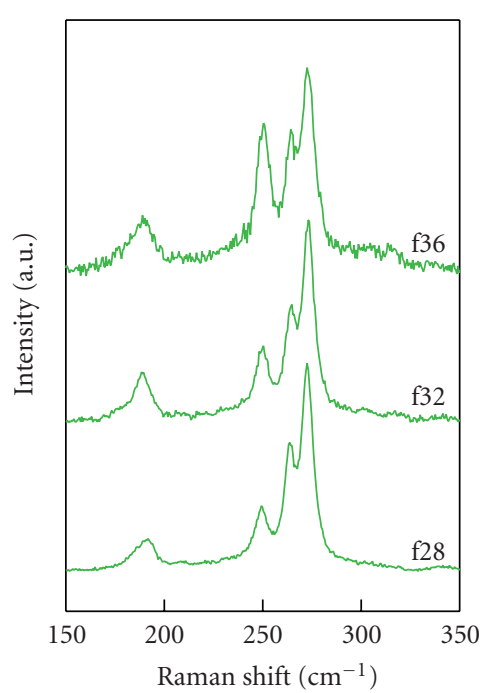

(b)

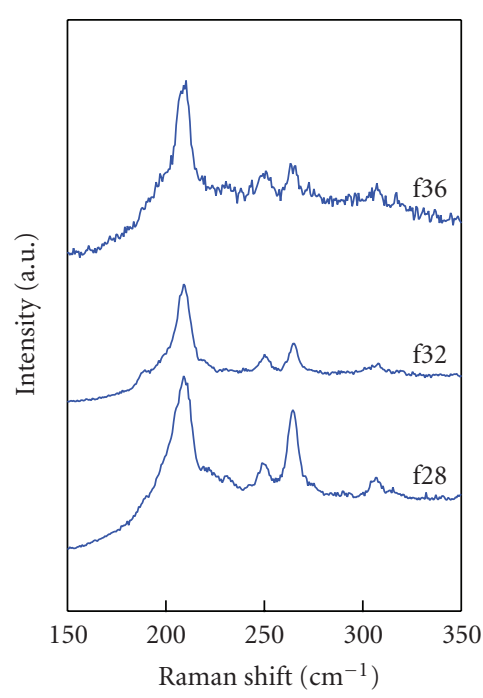

(c)

FIgURE 7: Raman spectra in the RBM region of three different fractions (f28, f32, and f36) of DNA-SWNTs (HiPco) with excitation wavelength of (a) 633, (b) 514, and (c) $488 \mathrm{~nm}$.

DNA-SWNTs and (b) DNA-DWNTs. Both PL maps show the presence of SWNTs/DWNTs having almost the same chiralities in narrow range. According to our previous PL study on DWNTs in reference to that of SWNTs [4], the majority of PL intensity of DWNTs stems from the inner carbon nanotubes rather than from the outer nanotubes. Similar to this study, we have found that there is no significant difference in the PL peak positions between SWNTs and DWNTs. In fact, we have not observed any shifts of PL peak positions irrespective of the CNTs length (30$400 \mathrm{~nm})$.

Sun et al. [19] reported, however, in their lengthdependent PL study on SWNTs that the blue shifts of the PL peaks are observed as the length $(60-7 \mathrm{~nm})$ of SWNTs decreases, which has been interpreted within the framework of the so-called finite length effects. They reported smaller peak shifts for the SWNTs of smaller diameter present in CoMoCAT samples.

At present, we think that the discrepancy is most probably due to the difference of the dispersion environment employed (i.e., DNA and surfactant) as well as the small length range investigated. Furthermore, since DNASWNTs/DWNTs show large red-shifts in PL peak positions with respect to the pristine SWNTs/DWNTs, the blue-shifts observed by Sun et al. in a different dispersion environment might be weakened in the present study. Further systematic studies are needed to clarify the length dependence of SWNTs and DWNTs on PL intensity and the peak positions.

The observed length dependence on the PL intensity for the SWNTs and DWNTs with several chiralities of $(6,5),(7$, $5)$, and $(8,3)$ is presented in Figure 4 . The observed general tendency is that the PL intensity decreases fairly rapidly as the length of SWNTs/DWNTs decreases. An almost linear relationship between the PL intensity and the nanotube length is clear, and this relationship is similarly observed both for SWNTs and DWNTs. A similar reduction of PL intensities for shortened SWNTs has been reported previously [23-25].

\subsection{Diameter Dependence on the Length Separated SWNTs}

3.2.1. Length Separation of DNA-SWNTs by High-Performance Liquid Chromatography. Figure 5 is a typical HPLC chromatogram of DNA-SWNTs (HiPco) by using Sepax CNT SEC columns. The detection UV wavelengths are 260 and $350 \mathrm{~nm}$. The overall HPLC pattern is almost the same as our previous report [9]. The elution of free DNA has a maximum at 46 minutes and that of DNA-SWNTs continues from 26 to 50 minutes.

Figure 6 shows histograms of the length distribution for fractions of $\mathrm{f} 28, \mathrm{f} 32$ and $\mathrm{f} 36$ (colored blue, green and red, respectively) based on the AFM measurements. The inserts show AFM images of HPLC fractions $\mathrm{f} 28, \mathrm{f} 32$ and $\mathrm{f} 36$. The width of the length distributions decreases progressively as the retention time increases. The average lengths of the hybrid materials contained in $\mathrm{f} 28, \mathrm{f} 32$ and $\mathrm{f} 36$ are found to be $353 \pm 104,170 \pm 26$ and $77 \pm 21 \mathrm{~nm}$, respectively, indicating that the present HPLC procedure is effective for separation of DNA-SWNTs by length.

3.2.2. Raman and Photoluminescence Measurements on DNASWNTs. Figure 7 shows the Raman spectra in radial breathing modes (RBMs) of DNA-SWNTs obtained from fractions of $\mathrm{f} 28, \mathrm{f} 32$, and $\mathrm{f} 36$ at 633,514 , and $488 \mathrm{~nm}$, respectively. The observed overall peak positions of the RBM are almost the same with each other, which suggests that these DNA-SWNTs have a similar diameter distribution.

Figure 8 shows PL contour plots of DNA-SWNTs of three different lengths as a function of emission and excitation wavelengths. The PL peaks are labeled by their assignment. The PL intensity gradually decreases as the fraction number 


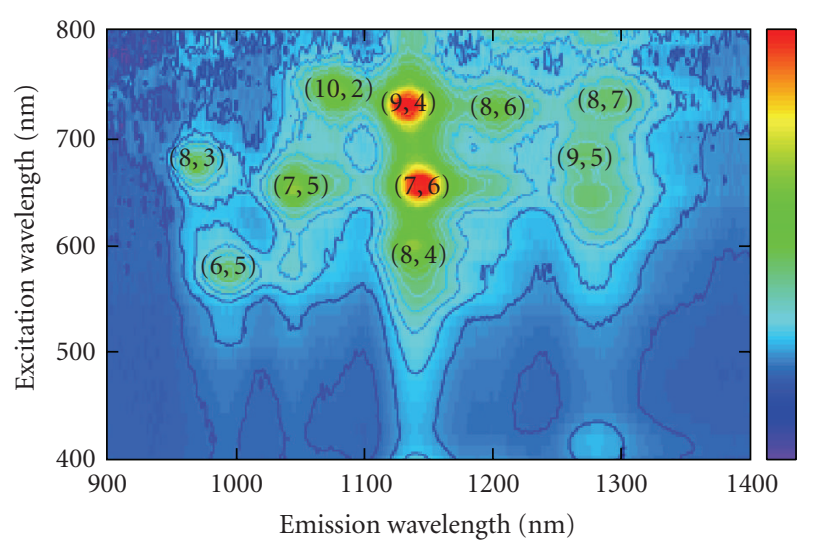

(a)

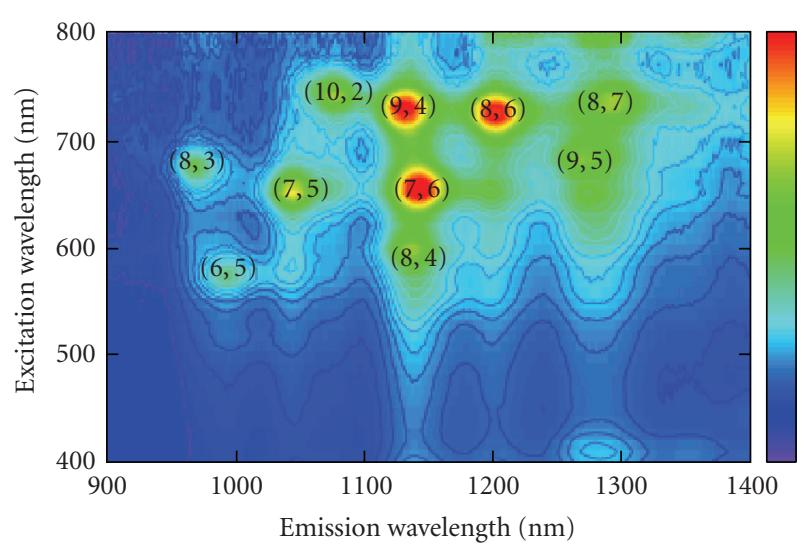

(b)

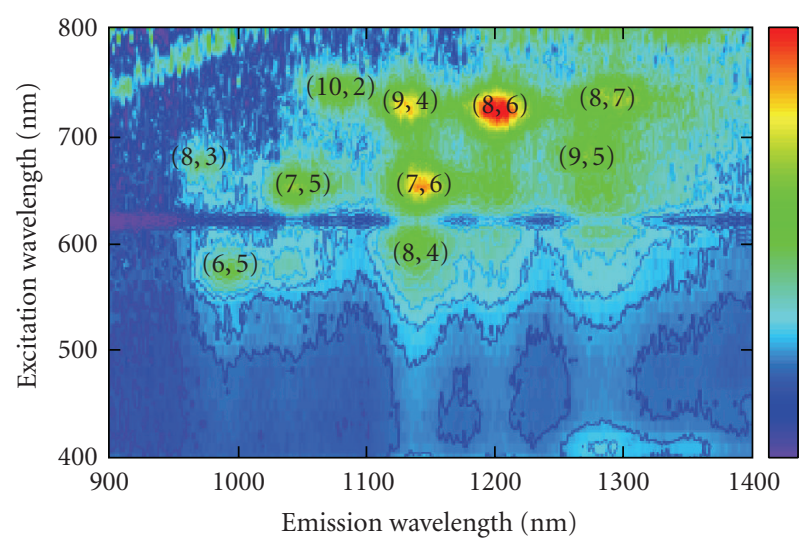

(c)

FIGURE 8: PL maps of DNA-SWNTs (HiPco) with fractions of (a) f28, (b) f32, and (c) f36.

increases (for shorter SWNTs). Also, the chirality distributions of DNA-SWNTs contained in each fraction differ from each other. For example, as seen from Figure 8, PL intensities of the SWNTs having chiralities of $(9,4),(7,6)$, and $(8,6)$ decrease rapidly as the length of the SWNTs decreases. A similar tendency for a sensitive change of the PL intensity on the SWNT length was reported by Arnold et al. [17].

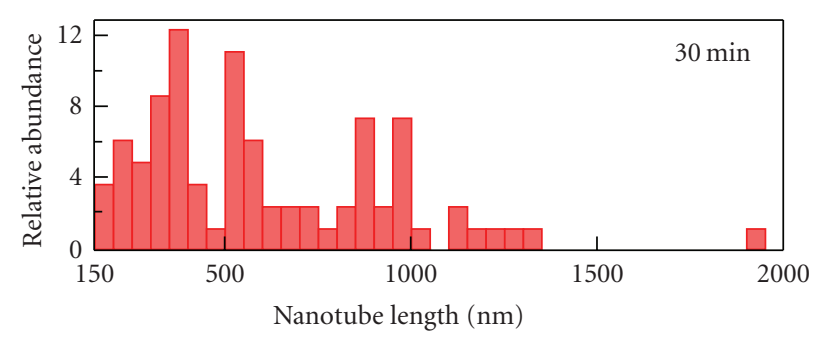

(a)

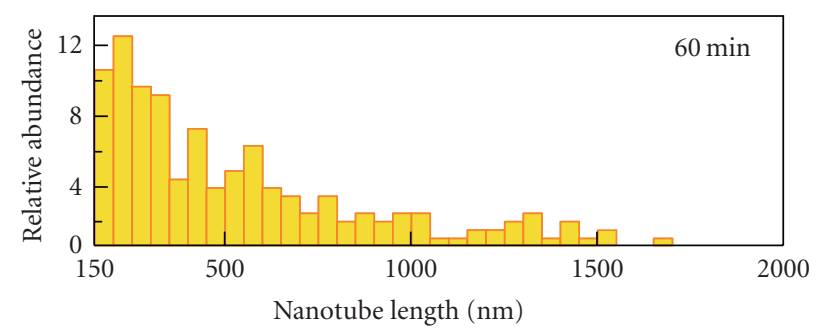

(b)

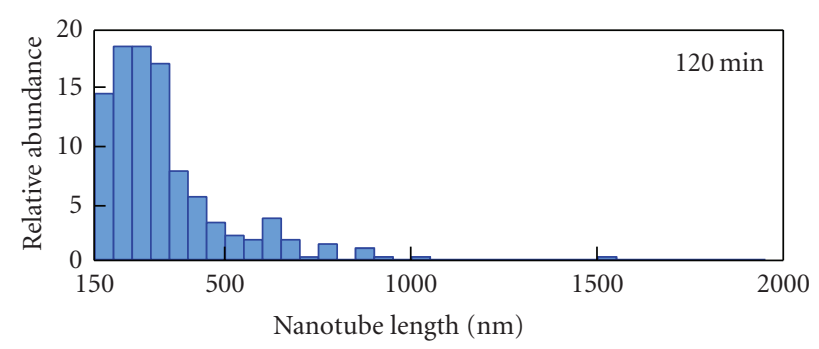

(c)

FIgURE 9: Histogram of length distribution of SDS-SWNTs (HiPco). The sonication times are 30 (red), 60 (yellow), and 120 minutes (blue).

According to the results of RBM measurements as shown in Figure 7, the diameter distributions of DNASWNTs contained in each fraction are similar with each other. However, the PL intensity distribution observed in the three fractions is differing with each other. These results strongly suggest that PL efficiency of the DNA-SWNTs varies sensitively depending not only on the chirality (or diameter) but similarly on the length of the hybrids.

3.2.3. SDS Dispersion of SWNTs. As described in the previous report, we have found that the length of the SWNTs depends on the duration of the sonication time. To further investigate the effect of SWNTs length on the PL intensity, SWNTs were dispersed with SDS at different sonication time. Since PL spectral features can be affected by the duration of sonication time of the SDS-SWNTs solution, $\mathrm{pH}$ of the solution was kept constant at $\mathrm{pH} 8.0$ by adding a proper amount of $\mathrm{NaOH}$ aq (in $\mathrm{D}_{2} \mathrm{O}$ ) during the PL measurements $[26,27]$.

Figure 9 shows histograms on the length of SDS-SWNTs at three different sonication times. To obtain information 


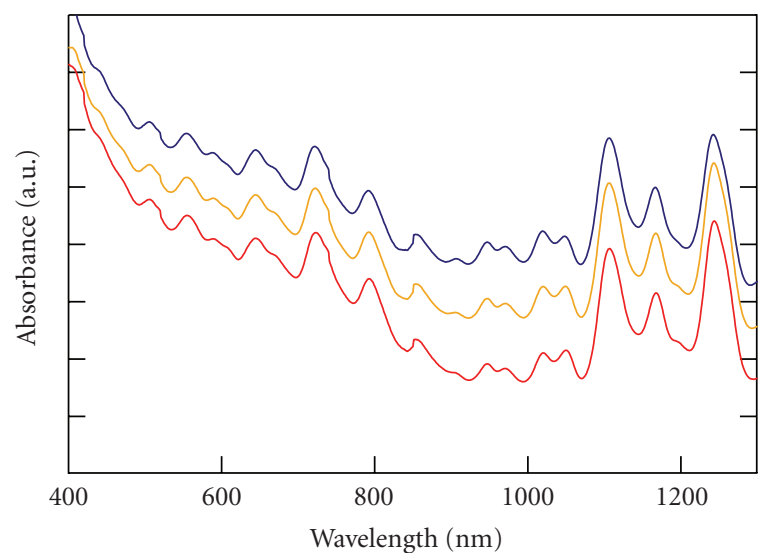

(a)

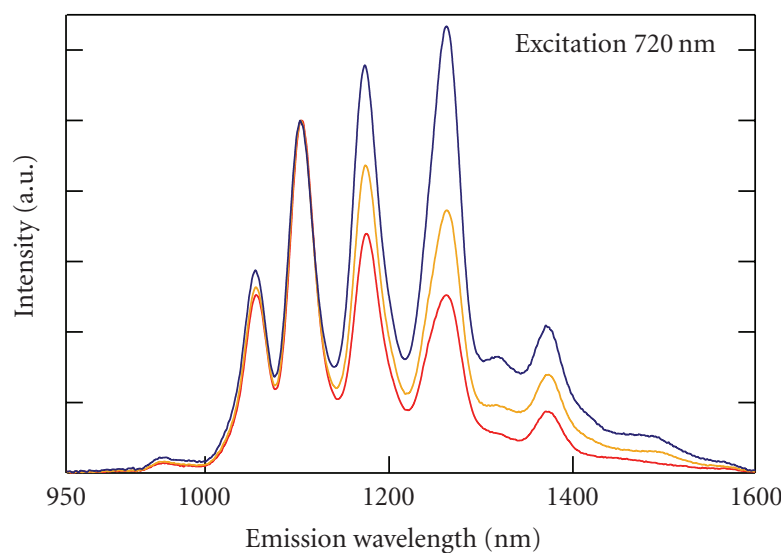

(b)

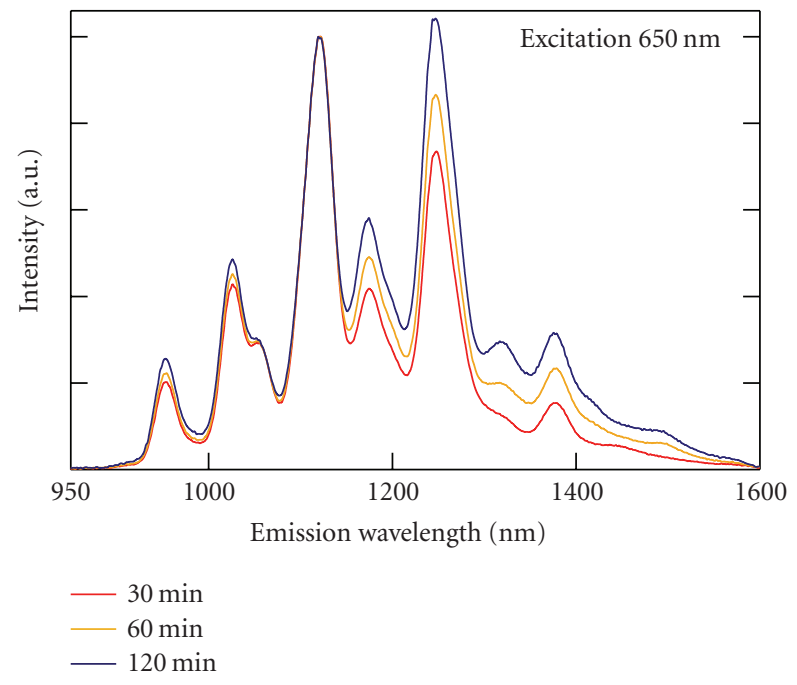

(c)

FIGURE 10: Spectra of SDS-SWNTs (HiPco) sonicated for 30 (red), 60 (yellow), and 120 minutes (blue). (a) UV-vis-NIR absorption. (b) and (c) PL spectra with excitation wavelength of 720 and $650 \mathrm{~nm}$, respectively.

on the length distributions, the number of SDS-SWNTs having the length longer than $150 \mathrm{~nm}$ was directly counted by AFM measurements. The length distribution of the hybrids decreases gradually as the sonication time increases similar to the DNA-SWNTs case described above.

Figure 10(a) shows UV-vis-NIR absorption spectra of SDS-SWNTs prepared by using three different sonication times, in which the overall spectral features are almost the same in the three spectra. Figures $10(\mathrm{~b})$ and $10(\mathrm{c})$ show the PL spectra of SDS-SWNTs prepared by three different sonication times at 720 and $650 \mathrm{~nm}$, respectively. PL spectra shown in Figures 10(b) and 10(c) are normalized at chiralities of $(10,2)$ and $(7,5)$, respectively. The relative PL intensities of thicker nanotubes show a gradual increase at longer sonication times (i.e., the average length of SDSSWNTs decreases). Namely, SDS-SWNTs show a similar dependence of PL intensities on the length of the SWNTs to that of the DNA-SWNTs case, indicating that such unique dependence might generally be observed regardless of the wrapping materials of SWNTs used for dispersion.

\section{Conclusions}

DNA-SWNTs and DNA-DWNTs have been separated well by length incorporating HPLC with newly developed sizeexclusion columns. The results of Raman, PL, and UVvis-NIR absorption measurements jointly suggest that PL intensity of the DNA-CNTs varies sensitively depending not only on the chirality (or diameter) but more importantly on the length of the CNTs. The present HPLC separation of DNA-SWNTs and -DWNTs has been proven to be a powerful method to obtain length-selected and water-soluble CNTs of high purity.

\section{Acknowledgments}

The authors thank Dr. M. Yoshikawa and Mr. K. Sato of Chemical Research Laboratories, Toray Industries, Inc. for preparation of DWNTs and Mr. Hirose of nacalai tesque, Inc. for the development of new SEC columns. This work has been supported by the Grant-in-Aids for Specific 
Area Research (no. 19084008) on Carbon Nanotube NanoElectronics and for Scientific Research A (no. 19205003) of MEXT, Japan, and partly by the Global COE Program in Chemistry, Nagoya University.

\section{References}

[1] J. H. Hafner, C. L. Cheung, T. H. Oosterkamp, and C. M. Lieber, "High-yield assembly of individual single-walled carbon nanotube tips for scanning probe microscopies," Journal of Physical Chemistry B, vol. 105, no. 4, pp. 743-746, 2001.

[2] J. Kong, N. R. Franklin, C. Zhou, et al., "Nanotube molecular wires as chemical sensors," Science, vol. 287, no. 5453, pp. 622$625,2000$.

[3] C. W. Zhou, J. Kong, E. Yenilmez, and H. J. Dai, "Modulated chemical doping of individual carbon nanotubes," Science, vol. 290, no. 5496, pp. 1552-1555, 2000.

[4] N. Kishi, S. Kikuchi, P. Ramesh, T. Sugai, Y. Watanabe, and H. Shinohara, "Enhanced photoluminescence from very thin double-wall carbon nanotubes synthesized by the zeoliteCCVD method," Journal of Physical Chemistry B, vol. 110, no. 49, pp. 24816-24821, 2006.

[5] P. Ramesh, T. Okazaki, R. Taniguchi, et al., "Selective chemical vapor deposition synthesis of double-wall carbon nanotubes on mesoporous silica," Journal of Physical Chemistry B, vol. 109, no. 3, pp. 1141-1147, 2005.

[6] T. Shimada, T. Sugai, Y. Ohno, et al., "Double-wall carbon nanotube field-effect transistors: ambipolar transport characteristics," Applied Physics Letters, vol. 84, no. 13, pp. 24122414, 2004.

[7] M. J. O’Connell, S. H. Bachilo, C. B. Huffman, et al., "Band gap fluorescence from individual single-walled carbon nanotubes," Science, vol. 297, no. 5581, pp. 593-596, 2002.

[8] H. Dohi, S. Kikuchi, S. Kuwahara, T. Sugai, and H. Shinohara, "Synthesis and spectroscopic characterization of single-wall carbon nanotubes wrapped by glycoconjugate polymer with bioactive sugars," Chemical Physics Letters, vol. 428, no. 1-3, pp. 98-101, 2006.

[9] Y. Asada, H. Dohi, S. Kuwahara, T. Sugai, R. Kitaura, and H. Shinohara, "Synthesis and spectroscopic characterization of salmon DNA-wrapped single-wall carbon nanotubes," Nano, vol. 2, no. 5, pp. 295-299, 2007.

[10] S. M. Bachilo, M. S. Strano, C. Kittrell, R. H. Hauge, R. E. Smalley, and R. B. Weisman, "Structure-assigned optical spectra of single-walled carbon nanotubes," Science, vol. 298, no. 5602, pp. 2361-2366, 2002.

[11] T. Umeyama, N. Kadota, N. Tezuka, Y. Matano, and H. Imahori, "Photoinduced energy transfer in composites of poly[ ( $\mathrm{p}$-phenylene-1,2-vinylene)-co-(p-phenylene-1,1vinylidene)] and single-walled carbon nanotubes," Chemical Physics Letters, vol. 444, no. 4-6, pp. 263-267, 2007.

[12] Y. Maeda, S. Kimura, M. Kanda, et al., "Large-scale separation of metallic and semiconducting single-walled carbon nanotubes," Journal of the American Chemical Society, vol. 127, no. 29, pp. 10287-10290, 2005.

[13] Y. Miyata, K. Yanagi, Y. Maniwa, and H. Kataura, "Highly stabilized conductivity of metallic single wall carbon nanotube thin films," Journal of Physical Chemistry C, vol. 112, no. 10, pp. 3591-3596, 2008.

[14] M. Zheng, A. Jagota, E. D. Semke, et al., "DNA-assisted dispersion and separation of carbon nanotubes," Nature Materials, vol. 2, no. 5, pp. 338-342, 2003.
[15] M. S. Arnold, S. I. Stupp, and M. C. Hersam, "Enrichment of single-walled carbon nanotubes by diameter in density gradients," Nano Letters, vol. 5, no. 4, pp. 713-718, 2005.

[16] S. J. Tans, A. R. M. Verschueren, and C. Dekker, "Roomtemperature transistor based on a single carbon nanotube," Nature, vol. 393, no. 6680, pp. 49-52, 1998.

[17] K. Arnold, F. Hennrich, R. Krupke, S. Lebedkin, and M. M. Kappes, "Length separation studies of single walled carbon nanotube dispersions," Physica Status Solidi B, vol. 243, no. 13, pp. 3073-3076, 2006.

[18] X. Y. Huang, R. S. McLean, and M. Zheng, "High-resolution length sorting and purification of DNA-wrapped carbon nanotubes by size-exclusion chromatography," Analytical Chemistry, vol. 77, no. 19, pp. 6225-6228, 2005.

[19] X. Sun, S. Zaric, D. Daranciang, et al., "Optical properties of ultrashort semiconducting single-walled carbon nanotube capsules down to sub-10 nm," Journal of the American Chemical Society, vol. 130, no. 20, pp. 6551-6555, 2008.

[20] N. Nakashima, S. Okuzono, H. Murakami, T. Nakai, and K. Yoshikawa, "DNA dissolves single-walled carbon nanotubes in water," Chemistry Letters, vol. 32, no. 5, pp. 456-457, 2003.

[21] P. Nikolaev, M. J. Bronikowski, R. K. Bradley, et al., "Gasphase catalytic growth of single-walled carbon nanotubes from carbon monoxide," Chemical Physics Letters, vol. 313, no. 1-2, pp. 91-97, 1999.

[22] S. M. Bachilo, L. Balzano, J. E. Herrera, F. Pompeo, D. E. Resasco, and R. B. Weisman, "Narrow (n,m)-distribution of single-walled carbon nanotubes grown using a solid supported catalyst," Journal of the American Chemical Society, vol. 125, no. 37, pp. 11186-11187, 2003.

[23] J. A. Fagan, J. R. Simpson, B. J. Bauer, et al., "Lengthdependent optical effects in single-wall carbon nanotubes," Journal of the American Chemical Society, vol. 129, no. 34, pp. 10607-10612, 2007.

[24] L. Cognet, D. A. Tsyboulski, J.-D. R. Rocha, C. D. Doyle, J. M. Tour, and R. B. Weisman, "Stepwise quenching of exciton fluorescence in carbon nanotubes by single-molecule reactions," Science, vol. 316, no. 5830, pp. 1465-1468, 2007.

[25] D. A. Heller, R. M. Mayrhofer, S. Baik, Y. V. Grinkova, M. L. Usrey, and M. S. Strano, "Concomitant length and diameter separation of single-walled carbon nanotubes," Journal of the American Chemical Society, vol. 126, no. 44, pp. 14567-14573, 2004.

[26] M. J. O'Connell, E. E. Eibergen, and S. K. Doorn, "Chiral selectivity in the charge-transfer bleaching of single-walled carbon-nanotube spectra," Nature Materials, vol. 4, no. 5, pp. 412-418, 2005.

[27] M. S. Strano, C. B. Huffman, V. C. Moore, et al., "Reversible, band-gap-selective protonation of single-walled carbon nanotubes in solution," Journal of Physical Chemistry B, vol. 107, no. 29, pp. 6979-6985, 2003. 

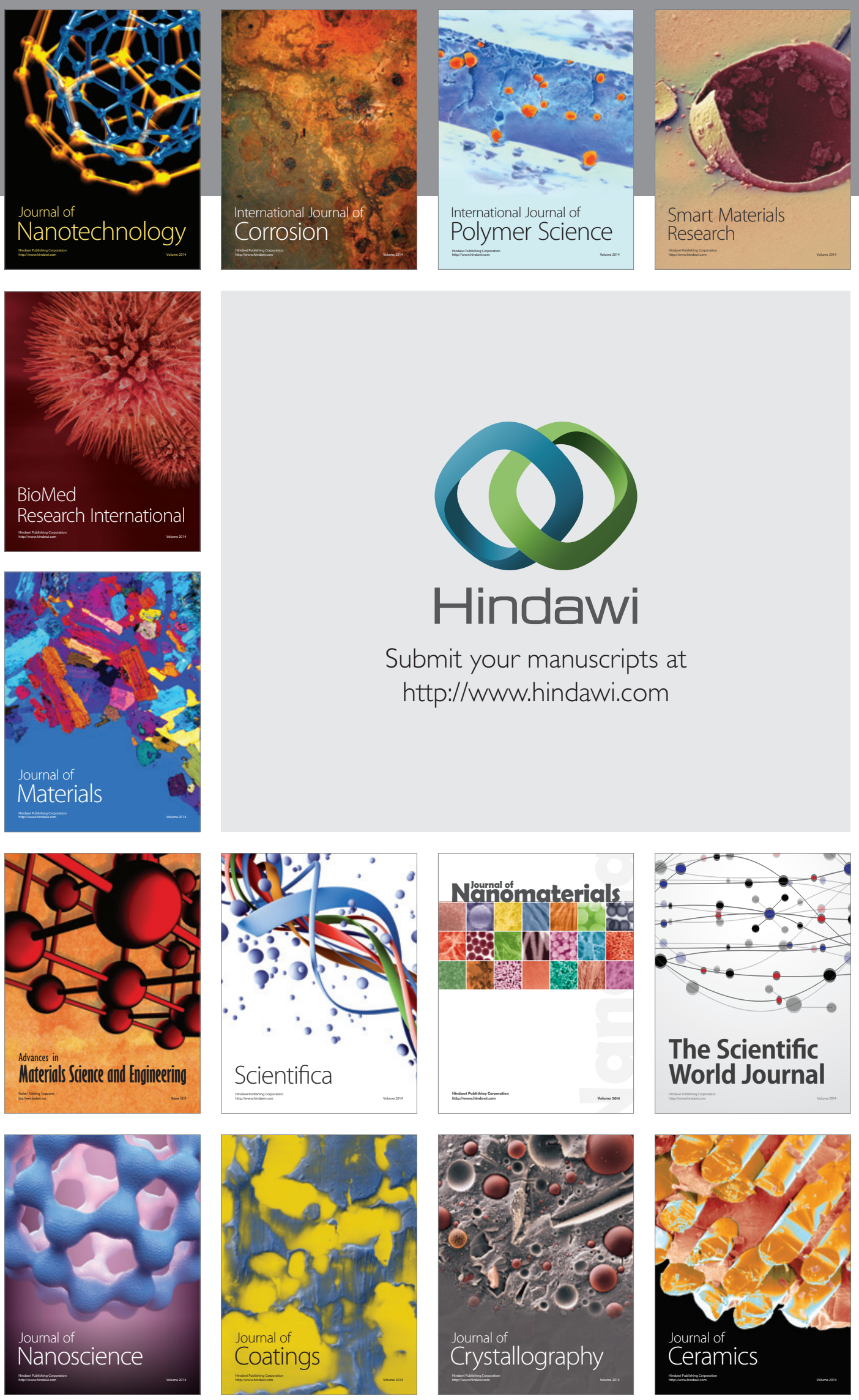

The Scientific World Journal

Submit your manuscripts at

http://www.hindawi.com

\section{World Journal}

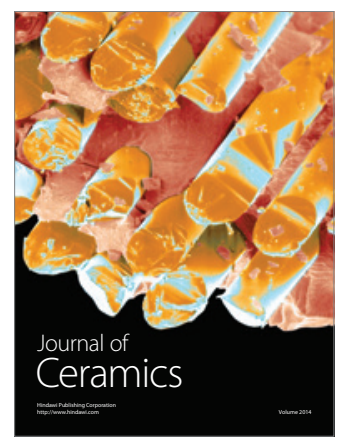

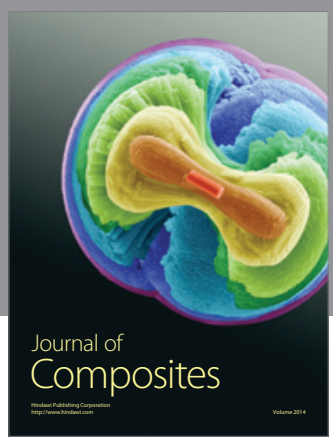
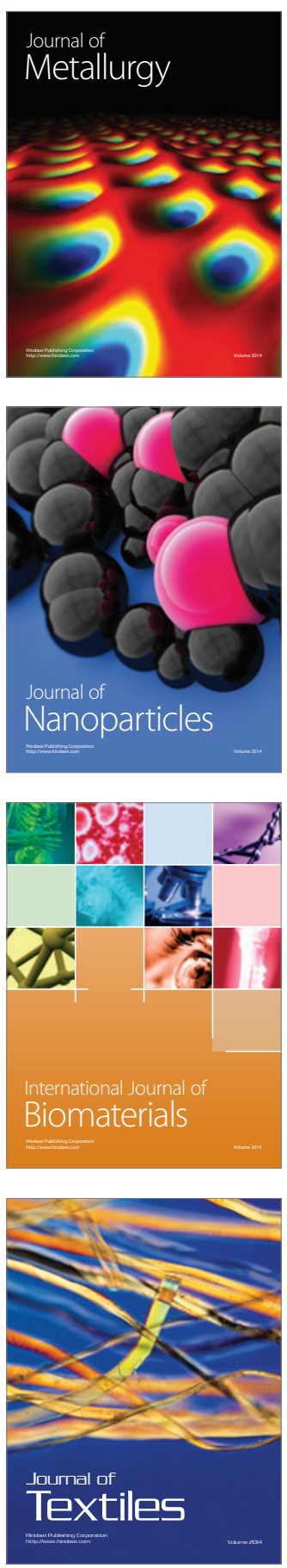